\title{
Peritonitis Due to Penicillium and Enterobacter in a Patient Receiving Continuous Ambulatory Peritoneal Dialysis
}

\author{
Maristela Böhlke', Person Antunes de Souza², Adriane Maria Delgado Menezes², \\ Juliana Martino Roth ${ }^{3}$ and Luiz Roberto Kramer ${ }^{1}$ \\ ${ }^{1}$ Catholic University of Pelotas; ${ }^{2}$ Federal University of Pelotas; ${ }^{3}$ Santa Casa de Pelotas Hospital; Pelotas, RS, Brazil
}

\begin{abstract}
Infectious peritonitis is a common complication of continuous ambulatory peritoneal dialysis (CAPD). Polymicrobial peritonitis accounts for about $8 \%$ of the peritonitis episodes and involves fungi in $9 \%$ to $33 \%$ of cases. We report the known first case of polymicrobial peritonitis in which Penicillium sp. was one of the organisms cultured and the ninth case of Penicillium peritonitis in CAPD.

Key-Words: Peritonitis, Penicillium, peritoneal dialysis.
\end{abstract}

Peritonitis is the main complication of peritoneal dialysis. The incidence rate of peritonitis depends on the end-stage renal disease population, varying from 0.5 episode/patient/ year [1] to 17 episodes/patient/year [2]. Most cases of peritonitis are caused by Gram-positive cocci, especially Staphylococcus epidermidis and Staphylococcus aureus [3]. Fungi are responsible for 9 to $14 \%$ of the cases, generally represented by Candida species [4,5]. Polymicrobial peritonitis accounts for about $8 \%$ of the peritonitis episodes [6-9] and involves fungal pathogen in 9 to $33 \%$ of cases $[6,10]$. Dialysis-related polymicrobial peritonitis usually occurs after multiple episodes of peritonitis ${ }^{8}$ and rarely is the result of an intra-abdominal process, such as perforation of an internal viscus [6-8]. Penicillium species of fungi are widely distributed in the environment and rarely cause human infection; the few cases described of invasive Penicillium infection involve immunocompromised patients or carriers of prosthetic devices, such as heart valves prosthesis or peritoneal catheters [11-13].

To the best of our knowledge, there have been only eight cases of Penicillium peritonitis in peritoneal dialysis patients previously reported in the English language literature [1218]. This report describes the ninth case, being the first in which the peritonitis is polymicrobial.

\section{Case Report}

A 46-year-old black female with end-stage renal disease receiving CAPD was admitted to this hospital in September 2003, with diffuse abdominal pain, nausea and vomiting. The dialysate was clear and showed fibrin. A specimen of peritoneal dialysis fluid was obtained for cell count and culture. She was empirically treated with intraperitoneal gentamicin and cephalotin. Her dialysate carried a white blood cell (WBC) count of $900 / \mathrm{mm}^{3}$ with $90 \%$ neutrophils and $10 \%$ lymphocytes. The empirical treatment was maintained for four days, when

Received on 15 August 2006; revised 16 November 2006.

Address for correspondence: Dr. Maristela Böhlke - Universidade Católica de Pelotas - 1054 Canoas st. - Zip code: 96090-130 - Pelotas - RS Brazil. Phone: 55-53-226-4098. E-mail: mbohlke@conesul.com.br.

The Brazilian Journal of Infectious Diseases 2007;11(1):166-168. (C) 2007 by The Brazilian Journal of Infectious Diseases and Contexto Publishing. All rights reserved. the culture was available, with growth of many colonies of a septate hyphae and Enterobacter aerogenes susceptible to gentamicin. The removal of the peritoneal catheter was proposed but the patient refused it. About 24 hours later, she became hypotensive, with episodes of hypoglycemia and altered mental status. The antibiotic regimen was shifted to intravenous imipenem (250 mg bid) and fluconazole (150 mg bid) and the patient was transferred to the Intensive Care Unit. A fungal smear from a new specimen of dialysate confirmed the presence of septate hyphae and fungal culture yielded many colonies of Penicillium sp.

Ultrasound examination did not detect any abnormality in the abdominal cavity. Over the course of 24 hours there was improvement in her clinical condition, with blood pressure increase after saline administration and improvement of mental status. The peritoneal effluent became progressively clearer, with reduction of WBC count to levels below $100 / \mathrm{mm}^{3}$. On admission day 19, she was discharged with oral fluconazole at a dose of $150 \mathrm{mg}$ daily for 14 days.

About 72 hours after discharge, the patient returned to the hospital complaining of abdominal pain, with clear dialysate. The peritoneal dialysis fluid cell count was $500 \mathrm{WBC} /$ $\mathrm{mm}^{3}$; Gram's stain, fungal smear and culture were negative. The patient was started on intravenous amphotericin B $(25 \mathrm{mg}$ bid) and imipenem (250 mg bid) and, 72 hours later, the catheter was removed. There was no bacterial or fungal growth in the catheter tip culture. Abdominal ultrasound was repeated and it did not show any structural abnormality. The patient evolved with aggravation of septicemia developing hemodynamics instability and died in November 2003.

\section{Past Medical History}

The patient had started hemodialysis in 1991. In 1993 she received a kidney transplant from a living related donor. There was graft loss three years later, when the patient returned to hemodialysis. After seven years she was transferred to peritoneal dialysis due to vascular access failure.

After five months on peritoneal dialysis, the patient presented tunnel infection with abscess formation, which was surgically drained. She was treated with endovenous vancomycin ( 1 gram every five days, 3 doses). The material from the abscess was not submitted to culture. The peritoneal effluent continued limpid. The tunnel infection was apparently 
Figure 1. Penicillium sp. monoverticillate culture stained with cotton blue, showed a dilatation in conidiophore(1) with phialides(2) and conidia chain(3) (100X).

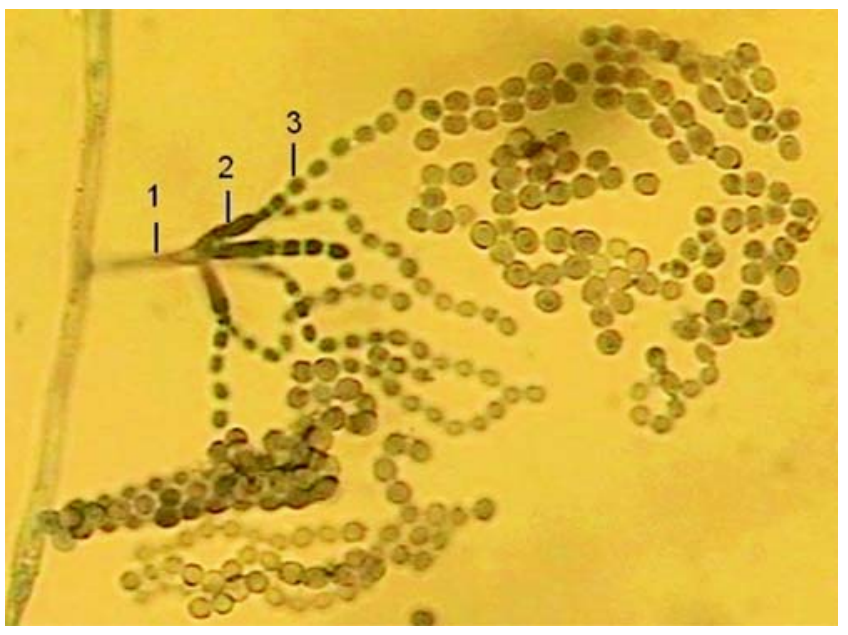

resolved and the patient remained asymptomatic until September 2003, when the case reported developed.

\section{Discussion}

The current report represents the ninth case described of Penicillium peritonitis in CAPD patient [12-18], in addition to representing the first case to occur as a polymicrobial infection. Dialysis related polymicrobial peritonitis usually occur after multiples episodes of peritonitis [8]. Furthermore, patients with polymicrobial peritonitis present higher rates of tunnel infection when compared to patients with singleorganism peritonitis. Holley et al. [6] describe an incidence of 0.28 tunnel infection/year in patients with polymicrobial peritonitis, versus 0.18 episode/year in patients with singleorganism peritonitis.

The traditional idea that the polymicrobial peritonitis is frequently associated with surgical abdominal pathologies comes from the inclusion of cases of bowel perforation from percutaneous placement of catheters in the early reports of peritonitis $^{6}$. However, series of cases described in the last years have shown that an enteric source accounts for only $7 \%$ of polymicrobial peritonitis [6-8]. Thus a laparotomy has been recommended just in situations that there is clinical suspicion of surgical pathology, being necessary in a small percentage of patients with polymicrobial peritonitis [19]. After repeated investigations by ultrasound, it was not found any intraabdominal surgical pathology in this patient. Besides, the Penicillium fungus does not belong to the normal intestinal flora, therefore, not being expected in a situation of visceral rupture. However, it is not possible to completely exclude the existence of gastrointestinal disease, which could explain the devastating course of infection in this case.

The late removal of the peritoneal catheter possibly contributed to the unfavorable outcome; in all the cases of Penicillium peritonitis previously described, there was patient recovery after antifungal therapy and early removal of the catheter [12-18]. However, the prognosis of polymicrobial peritonitis is poorer than single-organism peritonitis, with higher rates of transfer to hemodialysis or death.

In addition, it is important to have in mind that successfully treated cases have a better chance of being published.

Due to the small number of observations done so far, the most effective treatment for this type of infection is uncertain. It is believed that the best management is early catheter removal and administration of endovenous amphotericin $B_{12}$; however, there are reports of well-succeeded treatment with fluconazole $[13,16,18]$, despite the known resistance of the filamentous fungi to this therapeutic agent. The diagnosis of Penicillium peritonitis in CAPD can be difficult. The differentiation between fungal and bacterial peritonitis cannot be made clinically, either from signs and symptoms or from peritoneal WBC and differential counts [12]. The smear for fungus is little sensitive. Among the cases of Penicillium peritonitis described in the literature, a smear for fungus was positive in only two cases [12]. When the Penicillium is not identified by the smear but it is cultured in clinical laboratory condition, the fungi are often considerated a contaminant. In the present case, repeated isolation of Penicillium from the peritoneal fluid in a patient with peritonitis and sepsis indicate its pathogenic role.

In conclusion, Penicillium is not always a contaminant and should be considerated a pathogen in CAPD peritonitis. The diagnosis and the early catheter removal associated to antifungal treatment are fundamental for the effective management of this rare and potentially fatal infection.

\section{Acknowledgements}

We are in debt with Alice Carpena Carvalho for the translation of this article.

\section{References}

1. Rubin J.E., Marquardt E., Pierre M., Maxey R.W. Improved training techniques and UltraBag system resulted in lowered peritonitis rate in an inner-city population. Adv Perit Dial 1995;11:208-9.

2. Perera S., Palasuntheram C. Microbiological aspects of peritonitis in patients undergoing chronic peritoneal dialysis at the dialysis unit of Sri Jayawardenapura General Hospital. Ceylon Med J 2001;46:45-7.

3. Saklayen M.G. CAPD peritonitis. Incidence, pathogens, diagnosis, and management. Med Clin North Am 1990;74:997-1010.

4. Prasad K.N., Prasad N., Gupta A., et al. Fungal peritonitis in patients on continuous ambulatory peritoneal dialysis: a single center Indian experience. J Infect 2004;48(1):96-101.

5. Manzano-Gayosso P., Hernandez-Hernandez F., Mendez-Tovar L.J., et al. Fungal peritonitis in 15 patients on continuous ambulatory peritoneal dialysis (CAPD). Mycoses 2003;46(910):425-9.

6. Holley J.L., Bernardini J., Piraino B. Polymicrobial peritonitis in patients on continuous peritoneal dialysis. Am J Kidney Dis 1992;19(2):162-6.

7. Kiernan L., Finkelstein F.O., Kliger A.S., et al. Outcome of polymicrobial peritonitis in continuous ambulatory peritoneal dialysis patients. Am J Kidney Dis 1995;25(3):461-4. 
8. Kim G.C., Korbet S.M. Polymicrobial peritonitis in continuous peritoneal dialysis patients. Am J Kidney Dis 2000;36(5):1000-8.

9. Krishnan M., Thodis E., Ikonomopoulos D., et al. Predictors of outcome following bacterial peritonitis in peritoneal dialysis. Perit Dial Int 2002;22(5):573-81.

10. Digenis G.E., Abraham G., Savin E., et al. Peritonitis-related deaths in continuous ambulatory peritoneal dialysis (CAPD) patients. Perit Dial Int 1990;10:45-7.

11. Huang J.W., Chu T.S., Wu M.S., et al. Visible Penicillium spp. Colonization plaques on a Tenckhoff catheter without resultant peritonitis in a peritoneal dialysis patient. Nephrol Dial Transplant 2002;15:1872-3.

12. Hung Y.M., Chan H.H. Penicillium peritonitis in continuous ambulatory peritoneal dialysis. Dial Transplant 2003;32(11):720-3.

13. Hove M.G., Badalamenti J., Woods G.L. Penicillium peritonitis in a patient receiving continuous ambulatory peritoneal dialysis. Diagn Microbiol Infect Dis 1996;25:97-9.
14. Pearson J., McKinney T., Stone W. Penicillium peritonitis in a CAPD patient. Perit Dial Bull 1983;3:20-3.

15. Fahhoum J., Gelfand M.S. Peritonitis due to Penicillium sp. in a patient receiving continuous ambulatory peritoneal dialysis. South Med J 1996;89:87-8.

16. Qadir M.T., Cunha B.A. Penicillium peritonitis in a patient receiving continuous ambulatory peritoneal dialysis. Heart \& Lung 1998;27:67-8.

17. Equils O., Deville J.G., Shapiro A.M., Sanchez C.P. Penicillium peritonitis in an adolescent receiving chronic peritoneal dialysis. Pediatr Nephrol 1999;13:771-2.

18. Chang H.R., Shu K.H., Cheng C.H., et al. Peritoneal dialysisassociated Penicillium peritonitis. Am J Nephrol 2000;20:250-2.

19. Szeto C.C., Chow K.M., Wong T.Y., et al. Conservative management of polymicrobial peritonitis complicating peritoneal dialysis - a series of 140 consecutive cases. Am J Med 2002;113(9):728-33. 